\title{
Creative Contests: Knowledge Generation and Underlying Learning Dynamics for Idea Generation (An Abstract)
}

\author{
Nadia Steils and Salwa Hanine
}

\begin{abstract}
Crowdsourcing is nowadays considered as a promising tool for companies to benefit from the creativity of a crowd. Due to the heterogeneous nature of the crowd, one key question discussed by academics and practitioners is whether crowdsourcing contests can attract users who are skilled enough to make valuable contributions. In this context, this research aims to identify the dynamic learning processes underlying idea generation. Based on qualitative findings triangulating participants' and brands' perspectives through semi-structured interviews and secondary data, we identify six types of incoming and outgoing user skills. While previous research addressed four types of skills in the solution of problems, creative contests require and foster two additional types of skills that lead to more efficiency in ideation, namely, creative and social skills. Building on three types of brand instructions emerging from the data, findings show that value is added by the collectivity and two complementary dynamics generating valuable contributions and determining reparticipation in future contests: social learning and learning from experience. Our paper contributes to a better understanding of learning processes, types of skills, and brands' role in creative contests. From a managerial perspective, results provide guidance in designing crowdsourcing competitions by taking benefit from a multi-competent crowd.
\end{abstract}

\footnotetext{
N. Steils $(\bowtie)$

University of Namur, Namur, Belgium

IAE Lille, Lille, France

e-mail: nadia.steils@unamur.be

S. Hanine

Université de Nice Sophia Antipolis, GREDEG-CNRS, Nice, France

e-mail: salwahanine@gmail.com 